\title{
Effects of HSYA on the proliferation and apoptosis of MSCs exposed to hypoxic and serum deprivation conditions
}

\author{
XIAOQING SONG, LINING SU, HAIFENG YIN, JIN DAI and HUIPING WEI \\ Biology Office, Basic Medical College of Hebei North University, Zhangjiakou, Hebei 075000, P.R. China
}

Received October 14, 2017; Accepted March 5, 2018

DOI: $10.3892 /$ etm.2018.6125

\begin{abstract}
As a primary active ingredient of safflor yellow, hydroxysafflor yellow A (HSYA) exhibits notable antioxidative and neuroprotective effects. The aim of the present study was to investigate the protective effects of HSYA in mesenchymal stem cells (MSCs) exposed to hypoxia $\left(5 \% \mathrm{O}_{2}\right)$ and serum deprivation (H/SD), and to explore the mechanisms underlying HSYA-mediated protection. Under H/SD conditions, HSYA was applied to protect MSCs against injury. Cell viability, proliferation, apoptosis and reactive oxygen species (ROS) levels were determined using an 5-ethynyl-2'-deoxyuridine assay, MTT assay, Hoechst 33342/propidium iodide and 2',7'-dichlorodihydrofluorescein diacetate staining, respectively. The results revealed that $160 \mathrm{mg} / \mathrm{l} \mathrm{HSYA} \mathrm{significantly}$ reduced apoptosis and ROS levels compared with the H/SD group; however, HSYA demonstrated minimal effects on cell proliferation. A western blot assay demonstrated that HSYA reduced cleaved caspase-3 expression and cytC release from the mitochondria to the cytoplasm when compared with the H/SD group. In addition, western blotting and RT-qPCR analyses revealed that HSYA treatment significantly increased the expression of hypoxia inducible factor-1 $\alpha$ (HIF-1 $\alpha)$ and vascular endothelial growth factor (VEGF). In conclusion, the results of the current study demonstrated that HSYA exerts protective effects against H/SD-induced apoptosis in MSCs potentially via activation of the HIF-1 $\alpha /$ VEGF signaling pathway and stabilization of the mitochondrial membrane.
\end{abstract}

\section{Introduction}

Mesenchymal stem cells (MSCs) are a type of adult stem cell, distinguished by their characteristics of self-replication, low immunogenicity and easy isolation (1-3). Previous studies have demonstrated that MSCs have the potential to differentiate

Correspondence to: Professor Huiping Wei, Biology Office, Basic Medical College of Hebei North University, 11 South Diamond Road, Gaoxin, Zhangjiakou, Hebei 075000, P.R. China

E-mail:whp123456@sina.com

Key words: hydroxysafflor yellow A, mesenchymal stem cells, hypoxia, serum deprivation, proliferation, apoptosis into a number of different cell lineages under suitable conditions, including osteocytes (4), male germ-like cells (5) and diverse neuronal lineages $(1,6,7)$. Due to their distinctive plasticity, MSCs have been widely applied in tissue engineering to regenerate cells or tissues, and to repair damaged tissues. For instance, the intravenous administration of green fluorescent protein-labeled MSCs in vivo significantly improved neurological function in a rat stroke model via differentiation into neuron- and glial-like cells $(8,9)$. However, the therapeutic potential of MSCs is impeded by their poor survival rate following transplantation into lesion regions, where they are inevitably subjected to harsh microenvironments, including hypoxia, oligotrophy, peroxidation and inflammation (10). These environments eventually lead to a reduction or depletion of endogenous peroxidases and antioxidants (11). As a result, there is an abnormal increase in free radicals that cannot be scavenged, which induces irreversible cell apoptosis (11). Previous studies have demonstrated that the majority of transplanted MSCs die within a short time period and that the survival rate of human MSCs is $<0.44 \%$ at 4 days following transplantation into immunodeficient mice $(12,13)$. Furthermore, cellular damage induced by continual hypoxia weakens the regenerative capacity of MSCs (14). For instance, Deschepper et al (15) revealed that exposure to severe hypoxia $\left(1 \% \mathrm{O}_{2}\right)$ for 12 consecutive days led to the widespread death of MSCs; the number of viable MSCs was markedly reduced when MSCs were cultured in an environment containing $1 \% \mathrm{O}_{2}$ compared with $21 \% \mathrm{O}_{2}\left(2.5 \pm 1.2 \times 10^{4}\right.$ vs. $16 \pm 3 \times 10^{4}$ cells $)$. In addition, Zhu et al (10) demonstrated that hypoxia and serum deprivation $(\mathrm{H} / \mathrm{SD})$ treatment significantly upregulated the expression of caspase-3 in MSCs, which subsequently resulted in the initiation of the caspase-dependent apoptosis signaling pathway. These results demonstrate that serious abiotic stresses, such as H/SD, lead to MSC apoptosis. Therefore, if the tolerance of MSCs to adverse physiological and biochemical factors could be enhanced under hypoxic and ischemic conditions, this would be of great significance to their clinical application for the repair or regeneration of damaged tissues in vivo.

Currently, a number of researchers are aiming to develop reliable strategies that increase the survival of MSCs under adverse conditions in vitro. For instance, Lv et al (16) demonstrated that hypoxia-inducible factor (HIF)- $\alpha$ overexpression effectively promoted the viability of MSCs and suppressed apoptosis under hypoxic conditions. Chinese scholars have 
demonstrated that Chinese herbs and their active ingredients, including salidroside (17) and hydroxysafflor yellow A (HSYA) (18), may protect stem cells against apoptosis induced by toxic chemicals, including Cytarabine and $\beta$-mercaptoethanol. HSYA is a flavonoid extracted from the herb, Carthamus tinctorius L., which has been demonstrated to exhibit potent antioxidative effects on neurons (19) and endothelial cells (20) in vitro. To date, it has been extensively applied in the treatment of traumatic brain injury (21) and cardiac-cerebral vascular disease (22). A previous study demonstrated that the protective effects of HSYA against brain injury in a stroke animal model was achieved by attenuating the elevation of malondialdehyde, decreasing glutathione content and increasing superoxide dismutase activity (23).

To enhance the survival of MSCs in disease regions and promote the clinical application of these cells in tissue repair and regeneration, the authors of present study hypothesized that HSYA may protect MSCs against H/SD-induced apoptosis, thus improving the survival rate of transplanted MSCs. To investigate this hypothesis, H/SD treatment was utilized to mimic the unfavorable microenvironment of ischemic injury and the anti-apoptotic effects of HSYA on MSCs under H/SD conditions, and the associated signaling pathways were analyzed.

\section{Materials and methods}

MSC culture. A total of 6 male Sprague Dawley rats (3 weeks old; 45-50 g) were purchased from the Academy of Military Medical Science of China (Beijing, China), raised under the conditions of $20-25^{\circ} \mathrm{C}, 60 \pm 10 \%$ humidity and a $12: 12-\mathrm{h}$ light/dark cycle, and fed standard diet and provided with filtered water ad libitum. All animal experiments were approved by the Animal Ethics Committee of Hebei North University (Hebei, China; reference number: 2016-1-9-05). The isolation of MSCs from the 6 rats was performed in accordance with the National Institutes of Health Guide for the Care and Use of Laboratory Animals. Rats were anesthetized with an intraperitoneal injection of $400 \mathrm{mg} / \mathrm{kg}$ chloral hydrate before the rats were sacrificed and their femurs were dismembered. The bone marrow MSCs were then harvested from the femurs using complete Dulbecco's modified Eagle's medium containing 1 g/l glutamine (L-DMEM; Gibco; Thermo Fisher Scientific, Inc., Waltham, MA, USA) plus $10 \%$ fetal bovine serum (FBS; Gibco; Thermo Fisher Scientific, Inc.) and seeded into $25 \mathrm{~cm}^{2}$ culture flasks. The cells were maintained at $37^{\circ} \mathrm{C}$ in a humidified atmosphere with $95 \%$ air and $5 \% \mathrm{CO}_{2}$. When confluence had reached $\sim 90 \%$, cells were harvested with trypsin and subcultured until the third or fourth generation.

In order to observe the morphology of MSCs, Diff-Quick kit (Beijing Propbs Biotechnoloty Co., Ltd., Beijing, China) was applied to stain MSCs for three generations. In brief, MSCs were seeded in 24-well plate at a density of $1 \times 10^{4}$ and cultured under the aforementioned conditions. When the confluence reached $\sim 90 \%$, MSCs were washed three times using PBS with the $\mathrm{pH}$ at 7.2. Subsequently, at room temperature MSCs were fixed with Reagent A for $30 \mathrm{sec}$ and stained with Reagent B for $30 \mathrm{sec}$. Then MSCs were observed under a multifunctional fluorescence microscope (Eclipse 90i; Nikon
Corporation, Tokyo, Japan; magnification, x200) and images were captured.

Cell authentication. To confirm the mesenchymal properties of the cells cultured in the present study, cell-surface antigen profiles were determined by flow cytometry (FCM) analysis (BD Biosciences, Franklin Lakes, NJ, USA). MSCs express cluster of differentiation (CD)13, CD44, CD73, CD90 and CD105, but are negative for the expression of CD45, CD34, CD14 and CD19 (24). Therefore, third generation MSCs $\left(1 \times 10^{6}\right.$ cells $\left./ \mathrm{ml}\right)$ obtained in the present study were incubated with the following antibodies $(10 \mu 1)$ : Phycoerythrin (PE)-CD29 (2) (dilution, 1:1,000; cat. no. 562154; BD Pharmingen; BD Biosciences), allophycocyanin (APC)-CD90 (dilution, 1:1,000; cat. no. 561409; BD Pharmingen; BD Biosciences), fluorescein isothiocyanate (FITC)-CD34 (dilution, 1:1,000; cat. no. ab192547; Abcam, Cambridge, UK) and FITC-CD45 (dilution, 1:1,000; cat. no. 561867; BD Pharmingen; BD Biosciences) for $30 \mathrm{~min}$ at room temperature in the dark. This was followed by washing with PBS, and fluorescence was detected by FCM equipped with FACSDiva analytical software (version 8.0.1; BD Bioscience).

Cell treatments. In order to assess whether HSYA protects MSCs against H/SD-induced injury, H/SD treatment and HSYA protection were conducted on fourth generation MSCs. MSCs were first divided into normal, H/SD and protective groups. In the normal group, MSCs were cultured under the aforementioned conditions and regarded as the negative control. In the H/SD group, MSCs were cultured in L-DMEM (FBS-free) under hypoxic conditions $\left(5 \% \mathrm{O}_{2}\right)(25)$ for 6-48 h. In the protective group, MSCs were cultured for 6-48 h using L-DMEM (FBS-free) containing $160 \mathrm{mg} / 1 \mathrm{HSYA}$ (18) under hypoxic conditions.

Determination of cell apoptosis and reactive oxygen species (ROS) levels. Following the exposure of cells in each group to different culture conditions for $48 \mathrm{~h}$ at room temperature, double fluorescence staining using $0.5 \mu \mathrm{g} / \mathrm{ml}$ Hoechst 33342 and $50 \mu \mathrm{g} / \mathrm{ml}$ propidium iodide (PI) was employed to stain the nuclei for $10 \mathrm{~min}$ at room temperature to determine cell apoptosis. Hoechst 33342-positive cells are viable and PI-positive cells are apoptotic. Using a multifunctional fluorescence microscope (Nikon 90-I; Nikon Corporation, Tokyo, Japan), the percentage of apoptotic cells in each group was determined by counting Hoechst 33342- and PI-positive cells in 10 random fields of view.

The ROS level in MSCs was measured using an ROS assay kit (Beyotime Institute of Biotechnology, Haimen, China) in accordance with the manufacturer's protocol. After the exposure of MSCs in three groups to different culture conditions for 6, 12, 24 and 48 h, MSCs were incubated with 2',7'-dichlorodihydrofluorescein diacetate (DCF; Xiamen Bioluminor Bio-Tech Co., Ltd., Xiamen, China) for $30 \mathrm{~min}$ at $37^{\circ} \mathrm{C}$. Following washing with PBS three times, DCF fluorescence intensity was measured using FCM equipped with FACSDiva analytical software. Hoechst $33342(0.5 \mu \mathrm{g} / \mathrm{ml})$ was used to stain the nuclei after $24 \mathrm{~h}$ of treatment; Hoechst 33342 was incubated with the MSCs for $10 \mathrm{~min}$ at room temperature and images were obtained under a fluorescence microscope (magnification, x200). 
Cell viability and proliferation assay. Cell viability was assessed using an MTT assay kit (Shanghai Gefan Biotechnology Co., Ltd., Shanghai, China). Briefly, fourth generation MSCs $\left(8 \times 10^{3}\right.$ cells $\left./ \mathrm{ml}\right)$ were seeded into 96 -well plates. Once the cells had adhered to the culture vessel, they were divided into the aforementioned groups and exposed to the associated conditions for 6, 12, 24 and $48 \mathrm{~h}$. MTT reagent $(5 \mathrm{mg} / \mathrm{ml})$ was then added and the cells were incubated for $4 \mathrm{~h}$ at $37^{\circ} \mathrm{C}$. Ice-cold PBS was used to wash the cells, and $150 \mu 1 \mathrm{DMSO}$ was then added. The absorbance of each well was measured at $570 \mathrm{~nm}$ using a microplate reader (Bio-Rad Laboratories, Inc., Hercules, CA, USA). Each cell treatment was assayed $>3$ times.

For the determination of cell proliferation, a 5-ethynyl2'-deoxyuridine (EdU) kit (KeyGen Biotech, Co., Ltd., Nanjing, China,) was used. Briefly, $0.6 \mathrm{ml} /$ well MSCs at a density of $5 \times 10^{4}$ cells $/ \mathrm{ml}$ were seeded in 6 -well plates and when the MSCs had adhered to the surface of the culture vessel, MSCs were exposed to their respective culture conditions. EdU $(20 \mu \mathrm{M})$ was then added and the cells were incubated for $24 \mathrm{~h}$ at $37^{\circ} \mathrm{C}$. Thereafter, at room temperature the cells were washed with PBS, fixed in $4 \%$ polyformaldehyde for $10 \mathrm{~min}$ at room temperature and stained with kFluor488-azide [dilution, 3:1,000 (v/v); KeyGen Biotech, Co., Ltd.] for $30 \mathrm{~min}$ at room temperature. The cells were then stained with $0.5 \mu \mathrm{g} / \mathrm{ml}$ Hoechst for $10 \mathrm{~min}$ at room temperature. Images were acquired using a multifunctional fluorescence microscope. Three fields were randomly selected from each dish and at least three dishes from each group were counted to determine the number of EdU-positive cells.

Western blotting. Considering that HIF-1/vascular endothelial growth factor (VEGF) signaling contributes to protection against ischemic brain injury (26) and that caspase-3 and cytochrome c (cytC) promote cell apoptosis (27), a western blot analysis was performed to measure the expression of these proteins. MSCs $(0.6 \mathrm{ml} /$ well) were seeded in 6 -well plates at density of $5 \times 10^{4}$ cells $/ \mathrm{ml}$. When the cells were attached to the surface of culture plates, MSCs were exposed to the different treatment conditions for $24 \mathrm{~h}$. Total protein was extracted from MSCs on ice using RIPA Lysis buffer (Applygen Biotech, Co., Ltd., Beijing, China). Meanwhile, to detect the content of cytC in the cytoplasm, cytoplasmic protein was extracted from abovementioned MSCs using nuclear/cytoplasmic protein extraction kit (Sangon Biotech Co., Ltd., Shanghai, China). MSCs were harvested and treated with Buffer A for 15 min at room temperature, Buffer $\mathrm{B}$ was added for 1 min on ice and vortexed for $15 \mathrm{sec}$. The cells were then kept on ice for $1 \mathrm{~min}$ and centrifuged at $14,000 \mathrm{x} \mathrm{g}$ for $5 \mathrm{~min}$ at $4^{\circ} \mathrm{C}$. The resulting supernatant only contained cytoplasmic protein. The protein concentration was determined using a bicinchoninic acid assay kit (BestBio Company, Shanghai, China). Protein $(25 \mu \mathrm{g})$ from each sample was mixed with loading buffer and resolved by $11 \%$ SDS-PAGE.

Following electrophoresis, protein bands were electrotransferred onto polyvinylidene fluoride (PVDF) membranes. The membranes were blocked using $10 \%$ non-fat milk at room temperature for $1 \mathrm{~h}$ to prevent non-specific binding. The membranes were subsequently incubated with the following rabbit anti-rat antibodies obtained from Bioss Antibodies Inc.
(Beijing, China): HIF-1 $\alpha$ (dilution, 1:2,000; cat. no. bs-0737R), VEGF (dilution, 1:2,000; cat. no. bs-1665R), cleaved caspase-3 (dilution, 1:2,000; cat. no. bs-0081R), cytC (dilution, 1:2,000; cat. no. bs-0013R) and $\beta$-actin antibody (dilution, 1:2,000; cat. no. bs-0061R) at $37^{\circ} \mathrm{C}$ for $2 \mathrm{~h}$. Following washing with Tris-buffered saline and $0.1 \%$ Tween 20 , the membranes were incubated with horseradish peroxidase-conjugated goat anti-rabbit secondary antibody (dilution, 1:3,000; cat. no. bs-0295G; Bioss Antibodies Inc.) for $1 \mathrm{~h}$ at room temperature. An enhanced chemiluminescence detection reagent (Beyotime Institute of Biotechnology) was incubated with the PVDF membranes for 3-5 min at room temperature in the dark. The protein bands were visualized using the Aplegen Omega Lum W gel imaging system (Gel Company, Inc., San Francisco, CA, USA). The average area and band densities from 3-5 independent blots were used to represent the expression of all proteins. All experimental groups used a $\beta$-actin antibody as the loading control.

Reverse transcription quantitative polymerase chain reaction ( $R T-q P C R$ analysis). To further assess the potential protective effect of HSYA against H/SD-induced injury in MSCs, RT-qPCR was performed to assess the expression of HIF-1 $\alpha$ and VEGF following H/SD treatment. Total RNA was extracted from MSCs using TRNzol reagent (Tiangen Biotech Co., Ltd., Beijing, China) in accordance with the manufacturer's protocol. The purity of RNA was determined using spectrophotometry (wavelength range, 190-1,100 nm). The quality of extracted RNA was considered acceptable when the optical density (OD) at 260/280 nm was between 1.9 and 2.0. Total RNA $(2 \mu \mathrm{g})$ was then reverse transcribed into cDNA using the First-Strand cDNA Synthesis kit (Tiangen Biotech Co., Ltd.) containing oligo dT18 primers. The specific primers for RT-qPCR were designed using the online Primer-Blast tool (www.ncbi.nlm.nih.gov/tools/primer-blast/). Amplification was performed using the Premix Taq PCR kit (Takara Biotechnology Co., Ltd., Dalian, China), and the following thermocycling conditions: An initial denaturation step at $94^{\circ} \mathrm{C}$ for $5 \mathrm{~min}$, followed by 30 cycles of $94^{\circ} \mathrm{C}$ for $40 \mathrm{sec}, 55^{\circ} \mathrm{C}$ for $40 \mathrm{sec}$ and $72^{\circ} \mathrm{C}$ for $2 \mathrm{~min}$, and then one cycle at $72^{\circ} \mathrm{C}$ for $10 \mathrm{~min}$. The primers, amplification fragment lengths and annealing temperatures are presented in Table I. $\beta$-actin was used as a control housekeeping gene. The relative expression level of target genes was plotted as a fold change compared with the control using the $2^{-\Delta \Delta \mathrm{Cq}}$ method (28).

Statistical analysis. All data were analyzed using SPSS 17.0 software (SPSS, Inc., Chicago, IL, USA) and expressed as the mean \pm standard deviation. Homoscedasticity of primary data was first detected using Levene's test, and variance was demonstrated to be homogeneous. Comparisons among multiple groups were analyzed by two-way analysis of variance. Pairwise comparisons between different groups were performed using a Tukey's honest significance test. $\mathrm{P}<0.05$ was considered to indicate a statistically significant difference.

\section{Results}

Morphology and surface-antigen profile determination of MSCs. Following $72 \mathrm{~h}$ of primary culture, the majority 
Table I. Reverse transcription-quantitative polymerase chain reaction primer sequences.

\begin{tabular}{|c|c|c|c|}
\hline Genes & Primers $\left(5^{\prime}-3^{\prime}\right)$ & $\operatorname{Tm}\left({ }^{\circ} \mathrm{C}\right)$ & Fragment length (bp) \\
\hline HIF- $1 \alpha$ & & 55 & 218 \\
\hline Sense & AACAAACAGAATCTGTCCTCAAACC & & \\
\hline Antisense & CAGGTAATGGAGACATTGCCAG & & \\
\hline VEGF & & 55 & 151 \\
\hline Sense & CAGCGACAAGGCAGACTATT & & \\
\hline Antisense & GTTGGCACGATTTAAGAGGG & & \\
\hline$\beta$-actin & & 55 & 246 \\
\hline Sense & TCACCCACACTGTGCCCATCTATGA & & \\
\hline Antisense & CATCGGAACCGCTCATTGCCGATAG & & \\
\hline
\end{tabular}

HIF- $\alpha$, hypoxia-inducible factor- $\alpha$; VEGF, vascular endothelial growth factor; Tm, annealing temperature; bp, base pairs.
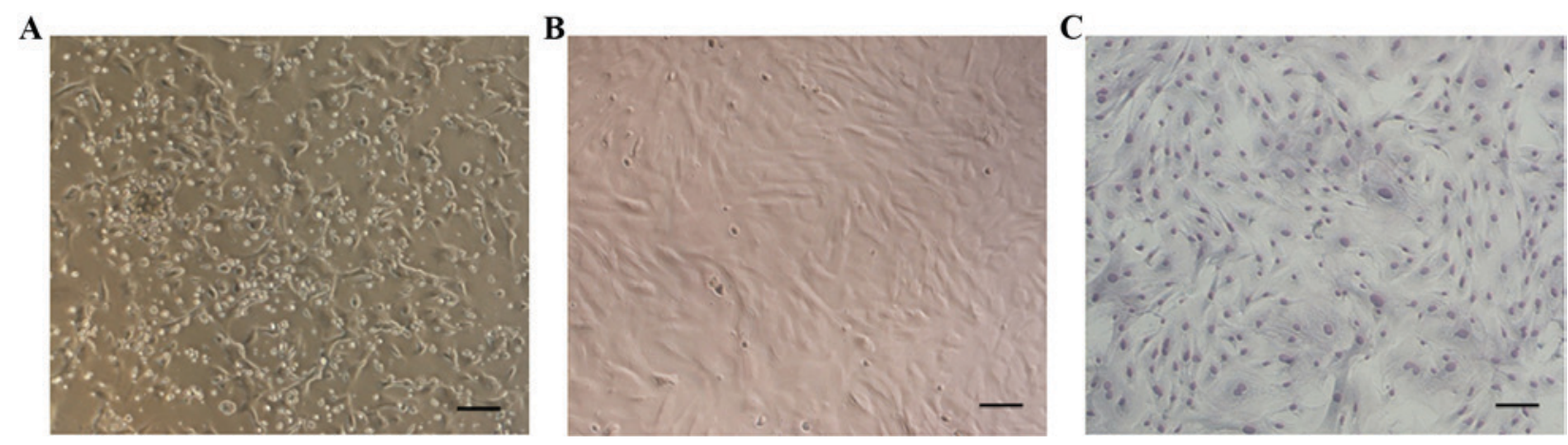

Figure 1. Morphological characteristics of MSCs. (A) The majority of cells were adherent following 3 days of primary culture. (B) Third generation MSCs exhibited a fibroblast- or spindle-like morphology. (C) Third generation MSCs were stained with Diff-Quick solution. Scale bar, $50 \mu \mathrm{m}$. MSCs, mesenchymal stem cells.

of adherent cells exhibited an elongated shape (Fig. 1A). Non-adherent cells were gradually removed by changing the medium. Following 7-8 days of culture, adherent cells reached $80-90 \%$ confluence. Cells were then harvested with trypsin and subcultured until the third generation. At this stage, the MSCs exhibited a fibroblast- or spindle-like morphology when they reached $80-90 \%$ confluence (Fig. 1B and C).

Third generation MSCs were incubated with FITC-conjugated antibodies targeting CD34 and CD45, a PE-conjugated antibody against CD29 and an APC-conjugated antibody targeting CD90. The cell surface-antigen profiles of MSCs were then determined by FCM. As demonstrated in Fig. 2, MSCs were strongly positive for CD29 and CD90, with positive rates of $97.05 \pm 2.38$ and $87.13 \pm 2.47 \%$, respectively. By contrast, MSCs were negative for CD34 and CD45, which were present in $1.94 \pm 0.27$ and $12.61 \pm 2.29 \%$ of the cell population, respectively (Fig. 2).

Detection of cell apoptosis and ROS levels. Staining with Hoechst 33342 and PI was used to detect the level of cell apoptosis. Compared with the normal group, a significant and time-dependent increase in the proportion of MSCs was observed following $\mathrm{H} / \mathrm{SD}$ treatment, and the proportion of apoptotic cells was $>35 \%$ in the H/SD group following $48 \mathrm{~h}$ of treatment (Fig. 3A and B). By contrast, the percentage of apoptotic cells was $\sim 15 \%$ in the protective group, which was significantly lower than the H/SD group. This indicated that HSYA treatment reduced the level of apoptosis.

An anoxic environment induces ROS production, which is one of the primary factors leading to cell apoptosis and senescence (11). As indicated in Fig. 3C-E, MSCs exposed to H/SD conditions exhibited a notable increase in ROS levels. The FCM results revealed that at $48 \mathrm{~h}$ following H/SD culture, a marked increase in DCF fluorescence intensity was observed, which was $\sim 6.5$-fold higher than the normal control group and $\sim 3$-fold higher than the protective group (Fig. 3C-E). As presented in Fig. 3E, fluorescence intensity at $48 \mathrm{~h}$ in the protective group was $221.33 \pm 20.21 \%$ and in the H/SD group it was $655.67 \pm 20.74 \%$.

To further elucidate the mechanisms underlying the anti-apoptotic effects exerted by HSYA, western blot analysis was performed to assess the expression levels of cleaved caspase-3 and cytoplasmic cytC. As presented in Fig. 3F and G, HSYA significantly suppressed the release of cytC from the mitochondria, as well as the expression of cleaved caspase-3 under $\mathrm{H} / \mathrm{SD}$ conditions. The relative band density of cytoplasmic cytC was $0.31 \pm 0.02$ in the protective group and $0.65 \pm 0.03$ in the $\mathrm{H} / \mathrm{SD}$ group, while the level of cleaved caspase- 3 was $0.29 \pm 0.02$ in the protective group and $0.59 \pm 0.06$ in the $\mathrm{H} / \mathrm{SD}$ group.

Cell proliferation and viability assays. To determine cell proliferation, medium containing an EdU/kFluor488-azide was 

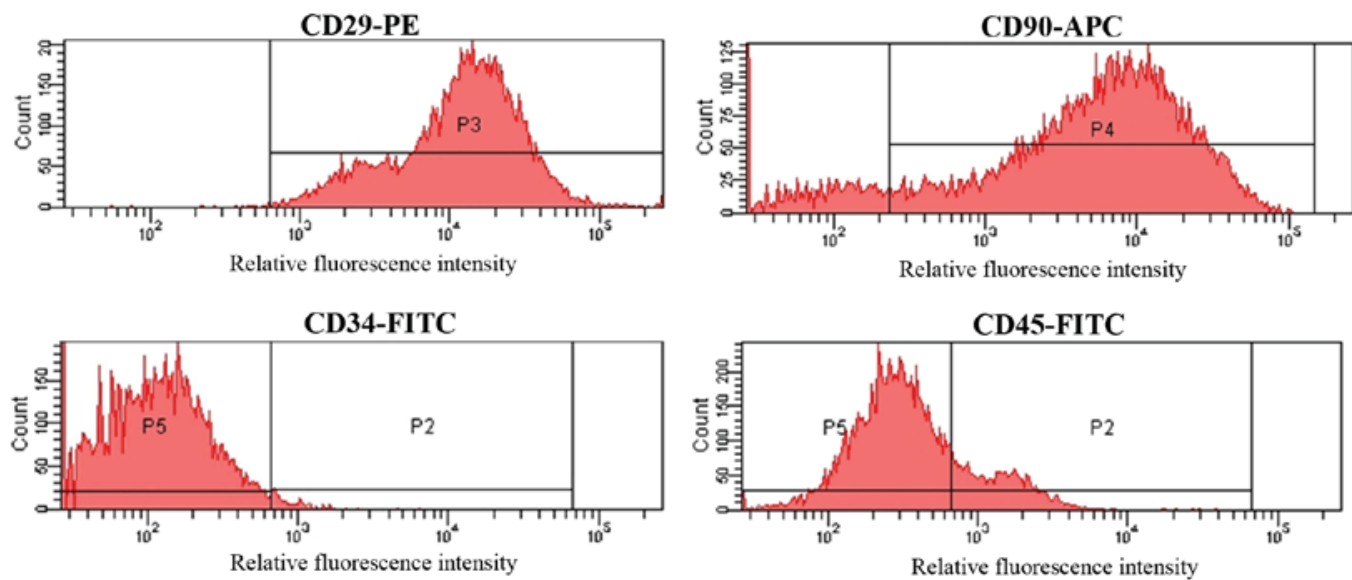

Figure 2. Determination of MSC surface antigens by FCM. MSCs were positive for CD29 and CD90 and negative for CD34 and CD45 by FCM analysis. P2-5 are the different gates that were assigned to distinguish cells. MSC, mesenchymal stem cells; FCM, flow cytometry; CD, cluster of differentiation; PE, phycoerythrin; APC, allophycocyanin; FITC, fluorescein isothiocyanate.
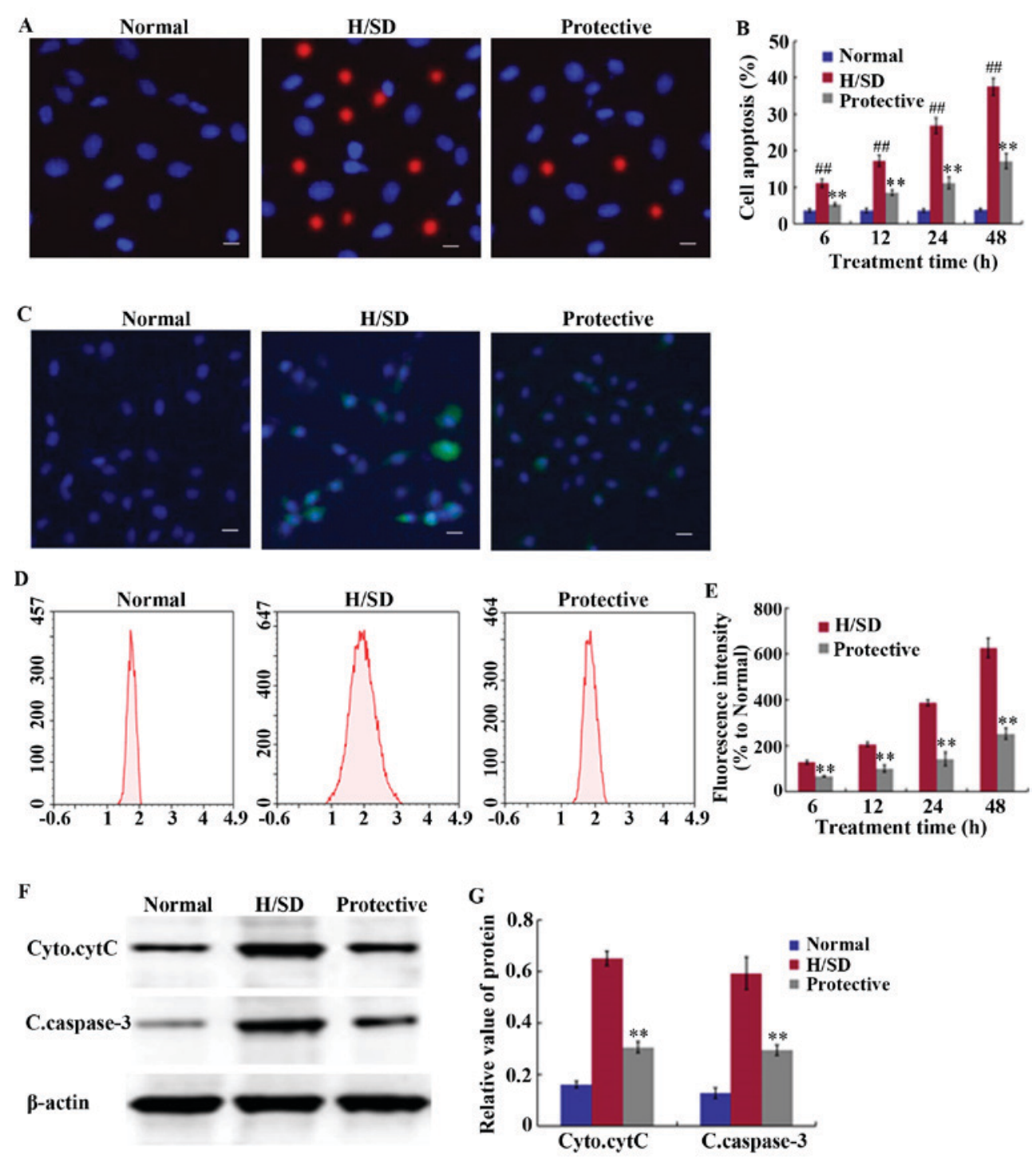

Figure 3. Effect of HSYA on apoptosis levels and ROS generation in MSCs. (A) Determination of cell apoptosis through fluorescence staining procedures following exposure to H/SD treatment for $48 \mathrm{~h}$ (scale bar, $50 \mu \mathrm{m}$ ). (B) Quantitative MSC apoptosis levels at 6,12, 24 and $48 \mathrm{~h}$. (C) Determination of ROS accumulation through fluorescence staining following exposure to H/SD treatment for $48 \mathrm{~h}$ (scale bar, $50 \mu \mathrm{m}$ ), and (D) determination of DCF fluorescence intensity by FCM expressed relative to the normal control group following 6-48 h of H/SD treatment. (E) Quantitative ROS levels at 6, 12, 24 and $48 \mathrm{~h}$. (F) Western blotting analysis of cytC and caspase-3 following $24 \mathrm{~h}$ of $\mathrm{H} / \mathrm{SD}$ culture. (G) Quantification of relative band intensities. ${ }^{\sharp \#} \mathrm{P}<0.01 \mathrm{vs}$. the normal. ${ }^{* *} \mathrm{P}<0.01 \mathrm{vs}$. the H/SD group. HSYA, hydroxysafflor yellow A; ROS, reactive oxygen species; MSCs, mesenchymal stem cells; H/SD, hypoxia and serum deprivation; FCM, flow cytometry; DCF, 2',7'-dichlorodihydrofluorescein diacetate; Cyto.cytC, cytoplasmic cytochrome C; C.caspsase-3, cleaved caspase-3. 
A

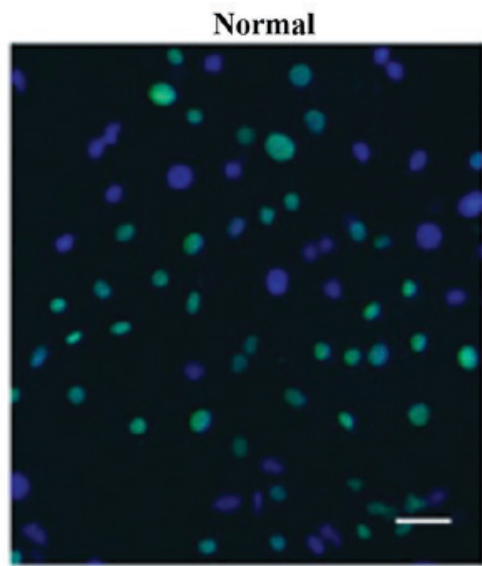

B

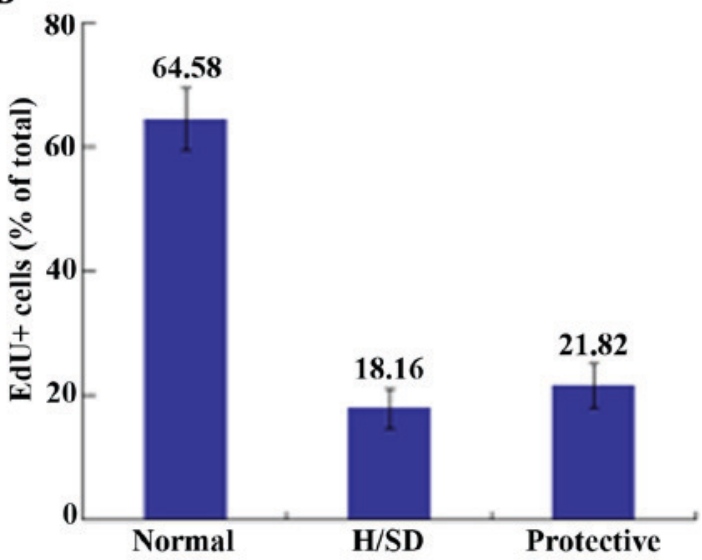

H/SD

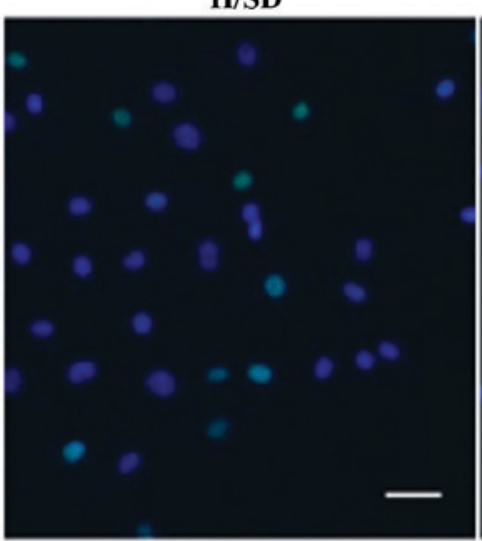

Protective

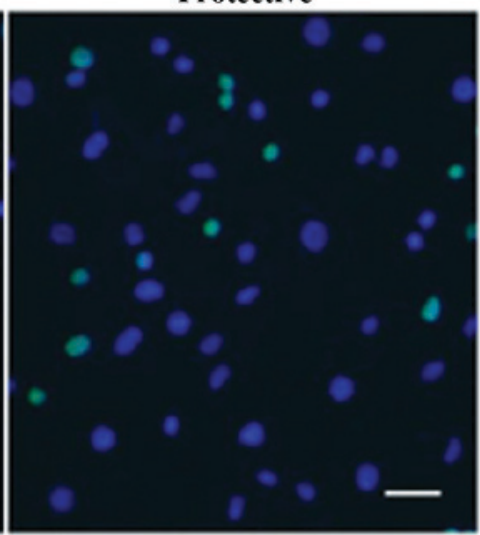

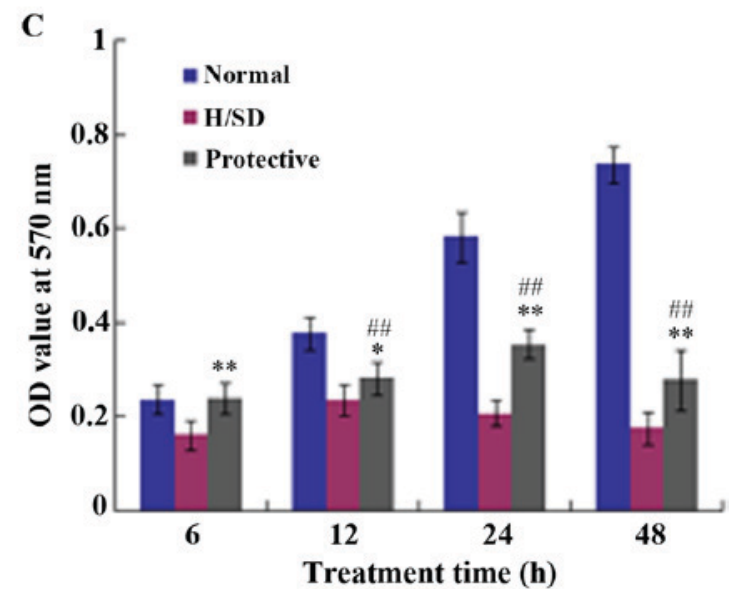

Figure 4. Assessment of MSC proliferation and viability under H/SD conditions. (A) Representative fluorescence microscopy images showing EdU staining of MSCs (Scale bar, $50 \mu \mathrm{m}$ ) and (B) the percentage of EdU-positive MSCs in each experimental group. (C) MTT assay indicating the viability of MSCs in each group following 6,12, 24 and $48 \mathrm{~h}$ exposure to normal $\mathrm{H} / \mathrm{SD}$ or protective conditions. ${ }^{\# \#} \mathrm{P}<0.01$ vs. the protective group. ${ }^{* *} \mathrm{P}<0.01 \mathrm{vs}$. the $\mathrm{H} / \mathrm{SD}$ group. MSCs, mesenchymal stem cells; H/SD, hypoxia and serum deprivation; EdU, 5-ethynyl-2'-deoxyuridine; OD, optical density.

applied to cell cultures for $24 \mathrm{~h}$. The percentage of EdU-positive cells in normal, $\mathrm{H} / \mathrm{SD}$ and protective groups was $64.58 \pm 5.02$, $18.16 \pm 3.19$ and $21.82 \pm 3.53 \%$, respectively (Fig. 4A and B). Thus, there was no marked difference between the H/SD and protective groups, which indicated that HSYA exhibited no notable effect on MSC proliferation under H/SD conditions.

An MTT assay was used to assess cell viability and the OD value was determined at $570 \mathrm{~nm}$ using a microplate reader. As demonstrated in Fig. 4C, cell viability in the protective HSYA group was significantly higher when compared with the H/SD group; however, it was reduced when compared with the normal group. Continuous H/SD treatment for $48 \mathrm{~h}$ led to a significant decrease in cell viability in the protective group compared with the normal group. As presented in Fig. 4C, cell viability in the protective group was $<40 \%$ of that in the normal group following $48 \mathrm{~h}$ of treatment. Therefore, compared with the H/SD group, HSYA protection exerted a positive effect on cell viability following a short period of exposure, which may be associated with ROS scavenging. The decrease of ROS stabilizes mitochondrial membrane integrity, which contributes to the maintenance of cell viability.

HIF-1/VEGF signaling pathway. To further investigate the anti-apoptotic effect of HSYA on MSCs under H/SD conditions, the potential underlying mechanisms were assessed. HIF-1 and its target gene VEGF are known to be involved in a number of biological functions, such as cell proliferation and anti-apoptotic mechanisms (29). Therefore, western blot and RT-qPCR analyses were employed in the present study to determine whether HSYA activates the HIF-1/VEGF signaling pathway. Western blotting indicated that the expression levels of HIF-1 $\alpha$ and VEGF in the protective group were significantly higher than those in the H/SD group following $24 \mathrm{~h}$ of H/SD treatment (Fig. 5A and B). Consistent with these observations, the RT-qPCR results demonstrated that 6-12 $\mathrm{h}$ of H/SD treatment upregulated the expression of HIF-1 $\alpha$ and VEGF in the H/SD group when compared with the normal group (Fig. 5C). H/SD exposure and HSYA protection for $24 \mathrm{~h}$ led to a peak in HIF-1 $\alpha$ and VEGF expression, after which their mRNA levels began to decrease. The decline in HIF-1 $\alpha$ and VEGF expression levels may have been associated with a decrease in cell viability.

\section{Discussion}

Due to their capacity for self-renewal and multipotency, MSCs have received attention for their use in developing treatments for central nervous system diseases, including cerebral 

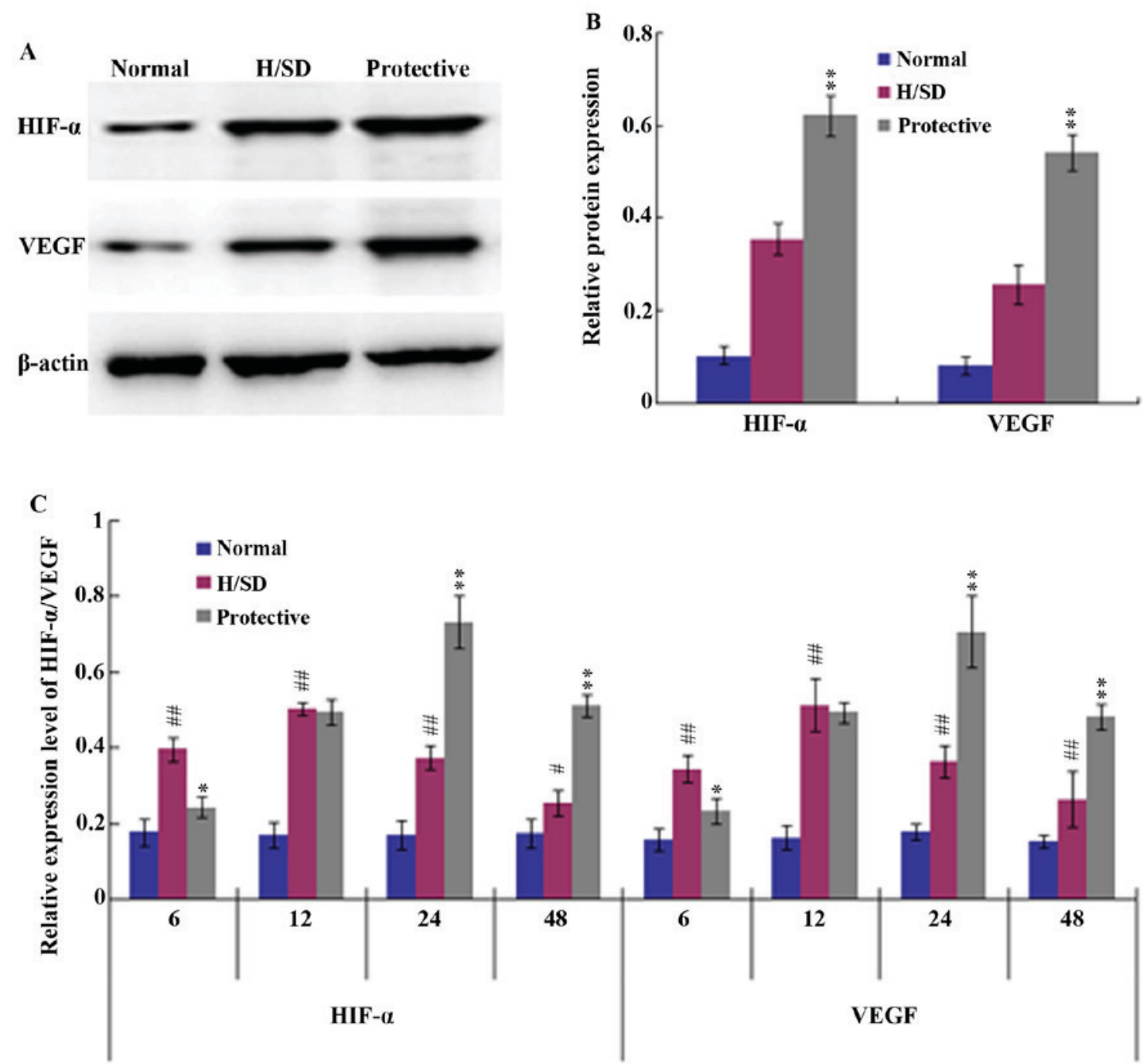

Figure 5. Expression of HIF- $\alpha$ and VEGF through western blot and RT-qPCR analyses. (A) Western blotting analysis and (B) quantitative analysis of band densities revealed that following MSC culture under H/SD conditions for $24 \mathrm{~h}, 160 \mathrm{mg} / \mathrm{l} \mathrm{HSYA}$ protection significantly increased the protein expression levels of HIF- $\alpha$ and VEGF. (C) RT-qPCR analysis demonstrated that HIF- $\alpha$ and VEGF mRNA levels were notably upregulated in HSYA protective group when compared with the normal and H/SD groups at 24 and $48 \mathrm{~h}$ of exposure. The results are presented as the mean \pm standard deviation of three separate experiments. ${ }^{~} \mathrm{P}<0.05$ and ${ }^{\# \#} \mathrm{P}<0.01$ vs. the normal group. ${ }^{*} \mathrm{P}<0.05$ and ${ }^{* *} \mathrm{P}<0.01$ vs. the $\mathrm{H} / \mathrm{SD}$ group. HIF- $\alpha$, hypoxia-inducible factor- $\alpha$; VEGF, vascular endothelial growth factor; RT-qPCR, reverse transcription quantitative polymerase chain reaction; MSC, mesenchymal stem cells; H/SD, hypoxia and serum deprivation; HSYA, hydroxysafflor yellow A.

ischemia (30), stroke (31) and spinal cord injury (32). MSCs have also been demonstrated to provide a suitable microenvironment for injured and damaged tissues through the secretion of cytokines and trophic factors (33), which serve important roles in the protection of injured neurons. However, MSCs transplanted into lesion sites are exposed to hypoxic and/or ischemic stress; thus, their curative potential is limited. Therefore, it is important to explore novel strategies for improving the survival rate of MSCs in vivo. Previous studies have demonstrated that the short-term exposure of MSCs to hypoxic conditions may promote cell proliferation and stemness, thus enriching the pool of cells potentially able to differentiate into multiple lineages (34-36). However, oxygen-derived free radicals induced by hypoxia are generally considered to be important contributors to MSC apoptosis (37). Recent studies have revealed that HSYA attenuates the breakdown of the blood brain barrier (26) and alleviates the imbalance between antioxidants and oxidants, leading to neuroprotective effects against ischemia-reperfusion injury in the spinal cord (38), liver (39) and brain (19) via the scavenging of free radicals.

In light of the neuroprotective effects conferred on cells or organs against oxidative damage, HSYA was applied to protect MSCs against H/SD-induced injury in the present study. The results demonstrated that when compared with the normal group, the apoptosis rate of MSCs exposed to H/SD significantly increased and $\sim 40 \%$ of MSCs died following $48 \mathrm{~h}$ of H/SD treatment. By contrast, prior protection with $160 \mathrm{mg} / \mathrm{l} \mathrm{HSYA}$ notably increased the survival rate of MSCs under H/SD conditions and the apoptosis level in the protective group was $\sim 15 \%$ at the same time point.

As a by-product of oxidative phosphorylation, a moderate quantity of ROS is necessary for cell survival, proliferation and promotion of longevity (40). However, in pathological sites, hypoxia-induced ischemia leads to an imbalance between the formation and scavenging of free radicals, which results in excess ROS (41). ROS accumulation leads to the release of the 
pro-apoptotic protein cytC into the cytoplasm from mitochondria and initiates the mitochondrial apoptotic pathway (42). Once cytC is released into the cytoplasm, it activates the expression of the downstream caspase apoptotic family (43). In this superfamily, cleaved caspase-3, a key mediator of apoptosis, performs the terminal steps of cell apoptosis and regulates the upstream induction of cell death (44). The results of the current study revealed that $\mathrm{H} / \mathrm{SD}$ induced the production of excess ROS and that this effect was suppressed by HSYA treatment. Western blot analysis further confirmed the anti-apoptotic effect of HSYA and revealed that HSYA notably suppressed the release of cytC into the cytoplasm, reducing the caspase cascade reaction.

It has been suggested that the ROS scavenging action of HSYA may contribute to the maintenance and integrity of the mitochondrial membrane and inhibition of the mitochondrial apoptosis signaling pathway (22). The pre-treatment with medicines is an effective measure for decreasing MSC apoptosis under malignant conditions in vitro $(17,45)$. For instance, the application of Nicorandil (45) significantly protects MSCs against apoptosis induced by H/SD for $9 \mathrm{~h}$. Comparatively, the results of the present study demonstrated that the anti-apoptotic effects of HSYA were more considerable, as the apoptosis rate of MSCs in the protective group was $<50 \%$ of the H/SD group.

Further experiments were performed to elucidate the molecular mechanisms associated with the regulation of apoptosis by HSYA. Specifically, the involvement of the HIF-1/VEGF signaling pathway in the anti-apoptotic activity of HSYA in MSCs was assessed. The HIF-1/VEGF pathway serves a vital role in promoting the survival of various cell types under hypoxic conditions $(16,29,46)$. HIF-1 is a heterodimeric protein composed of two subunits, HIF-1 $\alpha$ and HIF-1 $\beta$ (29). HIF-1 $\alpha$ is a functional subunit and belongs to the helix-loop-helix family of transcription factors (47). Its transcriptional activity is regulated by intracellular oxygen concentrations (46). Under hypoxic conditions, HIF-1 $\alpha$ persists and HIF- $1 \alpha / \beta$ is transported into the nucleus where it binds to HIF responsive elements and activates the expression of downstream target genes, such as erythrpoietin and VEGF, which are involved in energy metabolism and oxygen delivery (29). A previous study demonstrated that HIF-1 may serve as a neurotrophic and neuroprotective factor, which promotes MSC-mediated neurological and functional recovery in the ischemic brain (48). It is well established that VEGF, induced by HIF-1 $\alpha$, binds to its complementary receptors to initiate ERK-1/2 signaling and then activates the Akt pathway, resulting in cell proliferation, anti-apoptosis, anti-inflammation and angiogenesis (29,49-51). It has also been revealed that HIF-1 $\alpha$-induced VEGF expression in MSCs exerts an essential protective anti-ischemic effect on cardiomyocytes (52). Consistent with these observations, Zhong et al (53) observed a significant increase in the release of VEGF in HIF-1 $\alpha$-modified MSCs, which exerted a protective effect on neuron-like cells or neurons following ischemia in vitro and in vivo through the elevated expression of survivin: A member of the inhibitors of apoptosis protein family. Furthermore, VEGF has been demonstrated to function as a neuroprotective factor in motor neurons (54). For instance, VEGF released by MSCs reduced neuronal apoptosis and promoted neuronal proliferation in a rat stroke model (9). However, although HSYA protection was observed to significantly elevate the expression of HIF-1 $\alpha$ and VEGF under H/SD conditions in the present study, the proliferative ability of MSCs in the protective group was not enhanced, which may be associated with the lack of growth factors under serum deprivation. However, in terms of ROS scavenging activity, HSYA protection enhanced cell viability during short-term $\mathrm{H} / \mathrm{SD}$ treatment.

In conclusion, the results of the present study indicated that HSYA improved the survival rate of MSCs under H/SD conditions, which mimic the microenvironment of ischemic diseases. Under these conditions, the pro-survival and anti-apoptotic effects of HSYA may depend on the activation of the HIF-1/VEGF signaling pathway, the reduction of ROS production and the maintenance of mitochondrial membrane integrity.

\section{Acknowledgements}

The authors would like to thank all teachers of the Morphology Key Lab of Hebei North University.

\section{Funding}

The present study was funded by the Major Projection of Hebei North University (grant no. ZD201413).

\section{Availability of data and materials}

The datasets used and/or analyzed during the current study are available from the corresponding author on reasonable request.

\section{Authors' contributions}

XS, HY and HW mainly participated in the study design, writing and critical revision. XS, LS and JD mainly performed the experiments, and collected and analysed the data. All authors have read and approved this manuscript.

\section{Ethics approval and consent to participate}

All animal experiments were approved by the Animal Ethics Committee of Hebei North University (Hebei, China; reference no.: 2016-1-9-05).

\section{Consent for publication}

Not applicable.

\section{Competing interests}

The authors declare that they have no competing interests.

\section{References}

1. Kim EY, Lee KB, Yu J, Lee JH, Kim KJ, Han KW, Park KS, Lee DS and Kim MK: Neuronal cell differentiation of mesenchymal stem cells originating from canine amniotic fluid. Hum Cell 27: 51-58, 2014.

2. Jin W, Xu YP, Yang AH and Xing YQ: In vitro, induction and differentiation of umbilical cord mesenchymal stem cells into neuron-like cells by all-trans retinoic acid. Int J Ophthalmol 8: 250-256, 2015 . 
3. Han SM, Han SH, Coh YR, Jang G, Chan-Ra J, Kang SK, Lee HW and Youn HY: Enhanced proliferation and differentiation of Oct4- and Sox2-overexpressing human adipose tissue mesenchymal stem cells. Exp Mol Med 46: e101, 2014.

4. Heino TJ and Hentunen TA: Differentiation of osteoblasts and osteocytes from mesenchymal stem cells. Curr Stem cell Res Ther 3: 131-145, 2008

5. Nejad NA, Amidi F, Hoseini MA, Nia KN, Habibi M, Kajbafzadeh AM, Mazaheri Z and Yamini N: Male germ-like cell differentiation potential of human umbilical cord Wharton's jelly-derived mesenchymal stem cells in co-culture with human placenta cells in presence of BMP4 and retinoic acid. Iran J Basic Med Sci 18: 325-333, 2015.

6. Soleimani Mehranjani M and Chian MF: Cysteine: A novel neural inducer for rat bone marrow mesenchymal stem cells. Cel J 16: 195-202, 2014

7. Takeda YS and Xu Q: Neuronal differentiation of human mesenchymal stem cells using exosomes derived from differentiating neuronal cells. PLoS One 10: e0135111, 2015.

8. Wu J, Sun Z, Sun HS, Weisel RD, Keating A, Li ZH, Feng ZP and Li RK: Intravenously administered bone marrow cells migrate to damaged brain tissue and improve neural function in ischemic rats. Cell Transplant 16: 993-1005, 2008.

9. Deng YB, Ye WB, Hu ZZ, Yan Y, Wang Y, Takon BF, Zhou GQ and Zhou YF: Intravenously administered BMSCs reduce neuronal apoptosis and promote neuronal proliferation through the release of VEGF after stroke in rats. Neurol Res 32: 148-156, 2010 .

10. Zhu W, Chen J, Cong X, Hu S and Chen X: Hypoxia and serum deprivation-induced apoptosis in mesenchymal stem cells. Stem Cells 24: 416-425, 2006.

11. Nigam S and Schewe T: Phospholipase A(2)s and lipid peroxidation. Biochim Biophys Acta 1488: 167-181, 2000.

12. Tang YL, Tang Y, Zhang YC, Qian K, Shen L and Phillips MI: Improved graft mesenchymal stem cell survival in ischemic heart with a hypoxia-regulated heme oxygenase-1 vector. J Am Coll Cardiol 46: 1339-1350, 2005.

13. Toma C, Pittenger MF, Cahill KS, Byrne BJ and Kessler PD: Human mesenchymal stem cells differentiate to a cardiomyocyte phenotype in the adult murine heart. Circulation 105: 93-98, 2002.

14. Buravkova LB, Andreeva ER, Gogvadze V and Zhivotovsky B: Mesenchymal stem cells and hypoxia: Where are we? Mitochondrion 19: 105-112, 2014.

15. Deschepper M, Oudina K, David B, Myrtil C, Collet C, Bensidhoum M, Logeart-Avramogiou D and Petite H: Survival and function of mesenchymal stem cells (MSCs) depend on glucose to overcome exposure to long-term, severe and continuous hypoxia. J Cell Mol Med 15: 1505-1514, 2011.

16. Lv B, Li F, Fang J, Xu L, Sun C, Han J, Hua T, Zhang Z, Feng Z and Jiang $X$ : Hypoxia inducible factor $1 \alpha$ promotes survival of mesenchymal stem cells under hypoxia. Am J Transl Res 9 : 1521-1529, 2017.

17. Wei YP, Bai H, Sun YQ, Bao S, Qian R and Liu L: Effects of salidroside on apoptosis of bone marrow mesenchymal stem cells induced by Ara-C. Zhongguo Shi Yan Xue Ye Xue Za Zhi 21: 1572-1577, 2013 (In Chinese).

18. Song XQ, Su LN, Wei HP, Liu YH and Yin HF: Protective effects of hydroxysafflor yellow a against oxidative damage of $\beta$-mercaptoethanol during neural differentiation of mesenchymal stem cells. Chin Herbal Med 9: 282-288, 2017.

19. Wang X, Ma Z, Fu Z, Gao S, Yang L, Jin Y, Sun H, Wang C, Fan W, Chen L, et al: Hydroxysafflor yellow A protects neurons from excitotoxic death through inhibition of NMDARs. ASN Neuro 8: pii, 2016

20. Zhang L, Song Y, Liu CL, Zhu MC, Wang H, Liu K and Zhu HB Mechanism of hydroxysaf $\mathrm{f}$ lor yellow A induced endothelial cel proliferation. Chin Traditional Herbal Drugs 39: 403-408, 2008.

21. Wang Y, Zhang C, Peng W, Xia Z, Gan P, Huang W, Shi Y and Fan R: Hydroxysafflor yellow A exerts antioxidant effects in a rat model of traumatic brain injury. Mol Med Rep 14: 3690-3696, 2016.

22. Ramagiri S and Taliyan R: Neuroprotective effect of hydroxy safflor yellow A against cerebral Ischemi-reperfusion injury in rats: Putative role of mPTP. J Basic Clin Physiol Pharmacol 27: $1-8,2016$.

23. Wei X, Liu H, Sun X, Fu F, Zhang X, Wang J, An J and Ding H: Hydroxysafflor yellow A protects rat brains against ischemia-reperfusion injury by antioxidant action. Neurosci Lett 386: 58-62, 2005
24. Baksh D, Song L and Tuan RS: Adult mesenchymal stem cells: Characterization, differentiation, and application in cell and gene therapy. J Cell Mol Med 8: 301-316, 2004.

25. Ito A, Aoyama T, Yoshizawa M, Nagai M, Tajino J, Yamaguchi S, Lijima H, Zhang $X$ and Kuroki $\mathrm{H}$ : The effects of short-term hypoxia on human mesenchymal stem cell proliferation, viability and p16(INK4A) mRNA expression: Investigation using a simple hypoxic culture system with a deoxidizing agent. J Stem Cells Regen Med 11: 25-31, 2015.

26. Sun L, Yang L, Xu YW, Liang H, Han J, Zhao RJ and Cheng Y: Neuroprotection of hydroxysafflor yellow A in the transient focal ischemia: Inhibition of protein oxidation/nitration, 12/15-lipoxy-genase and blood-brain barrier disruption. Brain Res 1473: 227-235, 2012.

27. Zhang X, Sun JY, Gao MN and Li Y: Neuroprotection of overexpression of neuroglobin gene on injury of SH-SY5Y cells and relevant mechanism. Chin J Biologicals 26: 1768-1771, 2013.

28. Livak KJ and Schmittgen TD: Analysis of relative gene expression data using real-time quantitative PCR and the 2(-Delta Delta C(T)) method. Methods 25: 402-408, 2001

29. Cheng L, Yu H, Yan N, Lai K and Xiang M: Hypoxia-inducible factor-1 $\alpha$ target genes contribute to retinal neuroprotection. Front Cell Neurosci 11: 20, 2017.

30. Tian C, Wang X, Wang X, Wang L, Wang X, Wu S and Wan Z: Autologous bone marrow mesenchymal stem cell therapy in the subacute stage of traumatic brain injury by lumbar puncture. Exp Clin Transplant 11: 176-181, 2013.

31. Bhasin A, Srivastava MV, Mohanty S, Bhatia R, Kumaran SS and Bose S: Stem cell therapy: A clinical trial of stroke. Clin Neurol Neurosurg 115: 1003-1008, 2013.

32. Goni VG, Chhabra R, Gupta A, Marwaha N, Dhillon MS, Pebam S, Gopinathan NR and Kantharajanna SB: Safety profile, feasibility and early clinical outcome of co-transplantation of olfactory mucosa and bone marrow stem cells in chronic spinal cord injury patients. Asian Spine J 8: 484-490, 2014

33. Madrigal M, Rao KS and Riordan NH: A review of therapeutic effects of mesenchymal stem cell secretions and induction of secretory modification by different culture methods. J Trans Med 12: 260, 2014.

34. Fotia C, Massa A, Boriani F, Baldini N and Granchi D: Hypoxia enhances proliferation and stemness of human adipose-derived mesenchymal stem cells. Cytotechnology 67: 1073-1084, 2014.

35. Estrada JC, Albo C, Benguría A, Dopazo A, López-Romero P, Carrera-Quintanar L, Roche E, Clemente EP, Enríquez JA, Bernad A and Samper E: Culture of human mesenchymal stem cells at low oxygen tension improves growth and genetic stability by activating glycolysis. Cell Death Difer 19: 743-755, 2012.

36. Wang Y, Yang J, Li H, Wang X, Zhu L, Fan M and Wang X: Hypoxia promotes dopaminergic differentiation of mesenchymal stem cells and shows benefits for transplantation in a rat model of Parkinson's disease. PLoS One 8: e54296, 2013.

37. Chan PH: Role of oxidants in ischemic brain damage. Stroke 27 1124-1129, 1996

38. Shan LQ, Ma S, Qiu XC, Zhou Y, Zhang Y, Zheng LH, Ren PC, Wang YC, Fan QY and Ma BA: Hydroxysafflor yellow A protects spinal cords from ischemia/reperfusion injury in rabbits. BMC Neurosci 11: 98, 2010

39. Jiang S, Shi Z, Li C, Ma C, Bai X and Wang C: Hydroxysafflor yellow A attenuates ischemia/reperfusion-induced liver injury by suppressing macrophage activation. Int J Clin Exp Pathol 7: 2595-2608, 2014.

40. Acín-Pérez R, Carrascoso I, Baixauli F, Roche-Molina M, Latorre-Pellicer A, Fernández-Silva P, Mittelbrunn M, Sanchez-Madrid F, Pérez-Martos A, Lowell C, et al: ROS-triggered phosphorylation of complex II by Fgr kinase regulates cellular adaptation to fuel use. Cell Metab 19: 1020-1033, 2014.

41. Gao L, Liu R, Gao F, Wang Y, Jiang X and Gao X: Plasmon-mediated generation of reactive oxygen species from near-infrared light excited gold nanocages for photodynamic therapy in vitro. ACS Nano 8: 7260-7271, 2014

42. Dröse S, Brandt U and Wittig I: Mitochondrial respiratory chain complexes as sources and targets of thiol-based redox-regulation. Biochim Biophys Acta 1844: 1344-1354, 2014.

43. Zhang HY, Zhai L, Wang TT, LI S, Zhang YH and Guo YL: Picroside II plays a neuroprotective effect by inhibiting cyto C/caspase-9/caspase-3 signal pathway following ischemia/reperfusion injury in rats. Chin Pharmacol Bull 33: 668-674, 2017.

44. Kanwar JR, Kamalapuram SK and Kanwar RK: Survivin signaling in clinical oncology: A multifaceted dragon. Med Res Rev 33: 765-789, 2013 
45. Zhang F, Cui J, Lv B and Yu B: Nicorandil protects mesenchymal stem cells against hypoxia and serum deprivation-induced apoptosis. Int J Mol Med 36: 415-423, 2015

46. Gao J, Bai HJ, Wan F, Tian M, Li YY, Li YX and Si YC: Effects of ginsenoside on proliferation and differentiation of neural stem cells undergone oxygen-glucose deprivation/reperfusion. CJTCMP 32: 2291-2297, 2017.

47. Ejtehadifar M, Shamsasenjan K, Movassaghpour A Akbarzadehlaleh P, Dehdilani N, Abbasi P, Molaeipour Z and Saleh M: The effect of hypoxia on mesenchymal stem cell biology. Adv Pharm Bull 5: 141-149, 2015.

48. Vangeison G, Carr D, Federoff HJ and Rempe DA: The good, the bad, and the cell type-specific roles of hypoxia inducible factor-1 alpha in neurons and astrocytes. J Neurosci 28: 1988-1993, 2008.

49. Park IH, Kim KH, Choi HK, Shim JS, Whang SY, Hahn SJ, Kwon OJ and Oh IH: Constitutive stabilization of hypoxiainducible factor alpha selectively promotes the self-renewal of mesenchy-mal progenitors and maintains mesenchymal stromal cells in an undifferentiated state. Exp Mol Med 45: e44, 2013.

50. Ball SG, Shuttleworth CA and Kielty CM: Vascular endothelial growth factor can signal through platelet-derived growth factor receptors. J Cell Biol 177: 489-500, 2007.
51. Liao FL, Chen RL, Jiang S and Tian C: Effects of Notch signaling pathway on VEGF promoting Rat mesenchymal stem cells proliferation. Zhongguo Shi Yan Xue Ye Xue Za Zhi 22: 1068-1071, 2014 (In Chinese).

52. Dai Y, Xu M, Wang Y, Pasha Z, Li T and Ashraf M: HIF-1alpha induced-VEGF overexpression in bone marrow stem cells protects cardiomyocytes against ischemia. J Mol Cell Cardiol 42: 1036-1044, 2007.

53. Zhong Q, Zhou Y, Ye W, Cai T, Zhang X and Deng DY: Hypoxia-inducible factor 1- $\alpha$-AA-modified bone marrow stem cells protect PC12 cells from hypoxia-induced apoptosis, partially through VEGF/PI3K/Akt/FoxO1 pathway. Stem Cells Dev 21: 2703-2717, 2012.

54. Oosthuyse B, Moons L, Storkebaum E, Beck H, Nuyens D, Brusselmans K, Van-Dorpe J, Hellings P, Gorselink M, Heymans S, et al: Deletion of the hypoxia-response element in the vascular endothelial growth factor promoter causes motor neuron degeneration. Nat Genet 28: 131-138, 2001.

This work is licensed under a Creative Commons Attribution-NonCommercial-NoDerivatives 4.0 International (CC BY-NC-ND 4.0) License. 